Old Dominion University

ODU Digital Commons

Electrical \& Computer Engineering Faculty

Publications

Electrical \& Computer Engineering

1986

\title{
Faceting at the Silicon (100) Crystal-Melt Interface: Theory and Experiment
}

Uzi Landman

W.D. Luedtke

R.N. Barnett

C.L. Cleveland

M.W. Ribarsky

See next page for additional authors

Follow this and additional works at: https://digitalcommons.odu.edu/ece_fac_pubs

Part of the Electrical and Computer Engineering Commons

\section{Original Publication Citation}

Landman, U., Luedtke, W. D., Barnett, R. N., Cleveland, C. L., Ribarsky, M. W., Arnold, E., ... Khan, B. (1986). Faceting at the silicon (100) crystal-melt interface: Theory and experiment. Physical Review Letters, 56(2), 155-158. doi:10.1103/PhysRevLett.56.155

This Article is brought to you for free and open access by the Electrical \& Computer Engineering at ODU Digital Commons. It has been accepted for inclusion in Electrical \& Computer Engineering Faculty Publications by an authorized administrator of ODU Digital Commons. For more information, please contact digitalcommons@odu.edu. 


\section{Authors}

Uzi Landman, W.D. Luedtke, R.N. Barnett, C.L. Cleveland, M.W. Ribarsky, Emil Arnold, S. Ramesh, H. Baumgart, A. Martinez, and B. Khan 


\title{
Faceting at the Silicon (100) Crystal-Melt Interface: Theory and Experiment
}

\author{
Uzi Landman, W. D. Luedtke, R. N. Barnett, C. L. Cleveland, and M. W. Ribarsky \\ School of Physics, Georgia Institute of Technology, Atlanta, Georgia 30332 \\ and
}

Emil Arnold, S. Ramesh, H. Baumgart, A. Martinez, and B. Khan

Philips Laboratories, Briarcliff Manor, New York 10510

(Received 30 September 1985)

\begin{abstract}
Molecular-dynamics simulations and in situ experimental observations of the melting and equilibrium structure of the crystalline $\mathrm{Si}(100)$-melt interface are described. The equilibrium interface is structured, exhibiting facets established on (111) planes.
\end{abstract}

PACS numbers: $68.55 . \mathrm{Rt}, 64.70 . \mathrm{Dv}, 68.45 . \mathrm{Kg}$

The study of the solid-vapor and solid-liquid interfaces has attracted a recent surge of interest because of improved experimental and theoretical techniques for probing phenomena such as surface melting, surface roughening, and interface morphology. In particular, it has been suggested that the structure of the solidmelt interface during zone-melting recrystallization of silicon critically determines the generation of the observed networks of low-angle grain boundaries. ${ }^{1}$ Ample evidence exists that surfaces of crystalline silicon become faceted upon melting, and the solid-melt interface of a growing Si crystal establishes itself on the (111) crystal planes. ${ }^{2}$ Several models have been proposed to understand the solid-liquid interface morphology at equilibrium, notably the simple two-layer model of Jackson. ${ }^{3}$ Silicon marginally satisfies the Jackson criterion for facet formation. It could be expected that under appropriate conditions such material would exhibit facets both on solidification and in melting. In previous experimental ${ }^{4,5}$ and theoretical ${ }^{1}$ studies the silicon crystal-melt interface was investigated under nonequilibrium conditions during growth by laser-induced zone melting of silicon films on $\mathrm{SiO}_{2}$. In this Letter we report on the first theoretical microscopic simulations and experimental in situ observations of the melting and equilibrium structure of that interface, which provide evidence for a (111)-faceting transformation at the interface.

In our theoretical studies we have used the molecular dynamics method, ${ }^{6}$ which consists of a numerical solution of the equations of motion of a large ensemble of interacting particles on refined spatial and temporal scales. The structural, dynamical, and stability properties of homogenous phases and equilibrium interphase interfaces, as well as the kinetics and dynamics of phase transformations (such as melting and solidification), are governed by the various contributions to the total energy (or free energy, at finite temperatures). ${ }^{6 \mathrm{~b}}$ The potential energy of an interacting system of particles can be written in general as a sum of contributions of varying order in the number of par- ticles (one-body, two-body, ... terms). Because of the directional covalent bonding, characteristic of tetrahedral semiconductors, a model of the potential for these materials must go beyond the often-used pair interactions, via the inclusion of nonadditive, angledependent contributions (three-body and higher order). In our simulations we have employed optimized two- and three-body potentials, $V_{2}$ and $V_{3}$, respectively, which have yielded a rather adequate description of the structural properties of crystalline and liquid silicon?

$$
\begin{aligned}
& V_{2}\left(r_{i j}\right)=A\left[B r_{i j}^{-P}-1\right] g_{\beta}\left(r_{i j}\right), \\
& V_{3}\left(r_{i}, r_{j}, r_{k}\right)=v_{j i k}+v_{i j k}+v_{i k j}, \\
& v_{j i k}=\lambda g_{\gamma}\left(r_{i j}\right) g_{\gamma}\left(r_{i k}\right)\left[\cos \theta_{j i k}+\frac{1}{3}\right]^{2},
\end{aligned}
$$

where $r_{i j}$ is the distance between atoms $i$ and $j$, and $g_{\gamma}(r)=\exp [\gamma /(r-a)]$ for $r<a$ and vanishes for $r \geqslant a$. In Eqs. (1) $r$ is expressed in units of $\sigma$ $=0.20951 \mathrm{~nm}$, the unit of energy is $\epsilon=50 \mathrm{kcal} /$ mole, and that of temperature is $T=\epsilon / k_{\mathrm{B}}$ (to convert to $T$ in Kelvins multiply the reduced temperature by $\left.2.5173 \times 10^{4}\right) ; A=7.044556277, B=0.6022245584$, $P=4, a=1.8, \lambda=21, \beta=1$, and $\gamma=1.2$. The time unit, t.u., is $\sigma(m / \epsilon)^{1 / 2}=7.6634 \times 10^{-14} \mathrm{~s}$ and the integration time step $\Delta t$, with use of a fifth-order predictor-corrector algorithm, is taken to be $1.5 \times 10^{-2}$ t.u.; with this choice and a frequent updating of the interaction lists, the total energy is conserved to at least six significant figures. As seen from Eq. (1c), the three-body contribution to the potential energy vanishes for the perfect tetrahedral angle. Therefore, the liquid is characterized by a higher magnitude of $V_{3}$ than the solid. While improvements to the potential functions are desirable, their general form and the degree of agreement with observed data which they provide $^{7}$ warrant their use in our study of the interface between condensed phases, i.e., at the solid-melt interface (conduction-electron-density-dependent potential-energy terms could be incorporated in future studies in a manner similar to that proposed by us recent- 
ly). ${ }^{8}$ Additionally, the molecular dynamics technique which we use allows for dynamical variations in particle density and structural changes via the Ansatz Lagrangean of Parrinello and Rahman, ${ }^{9}$ extended to include three-body interactions and planar $2 \mathrm{D}$ periodic boundary conditions.

Since zone-melted Si films on $\mathrm{SiO}_{2}$ tend to recrystallize with (100) texture, ${ }^{4,5}$ we start with a silicon crystal consisting of $N_{L}$ dynamic layers, with $N_{P}$ particles per layer, exposing the (001) face. The $z$ axis is taken parallel to the [001] direction, and the 2D cell is defined by the [110] and [110] directions. The bottom layer of the crystal (layer number 1) is positioned in contact with a static silicon substrate. Simulations for two systems were performed: (i) $N_{L}=28$ and $N_{P}$ $=36$, for which results are shown, and (ii) $N_{L}=24$ and $N_{P}=144$, yielding similar results. The $N_{L}$ values were chosen so as to minimize the static substrate effects at the interface.

Following equilibration of the total system at a temperature $T=0.064$ and zero external pressure, a portion of the system (about $N_{L} / 2$ from the top, free surface) was heated via scaling of particle velocities. During the subsequent dynamical evolution towards equilibrium, melting initiated, with the melting front propagating from the exposed surface towards the bulk, exhibiting a tendency for facet formation upon melting. After a prolonged period (for the data presented below, runs in excess of $1.5 \times 10^{5} \Delta t$ ) an equilibrium crystal-melt coexistence was established, at an average kinetic temperature $T_{m}=0.0662$ \pm 0.0016 , uniform throughout the system. This value may be compared to the experimental melting temperature of silicon, $T_{m}=0.0669 \equiv 1683 \mathrm{~K}$. The equilibrium crystal-melt interface exhibits a pronounced structure, demonstrated by the sample particle trajectories, shown in Fig. 1(a). These are recorded for $2000 \Delta t$ and viewed along the $[\overline{1} 10]$ direction (denoted by a circle at the bottom left), where the breakup into alternating (111) and (111) crystalline planes is indicated. The melt region in the vicinity of the solid (facet) planes exhibits a certain degree of ordering due to the crystalline potential, resulting in a diffuseness of the interface at that region. ${ }^{6 \mathrm{~b}}$ To complement the picture, we show in Fig. 1(b) particle trajectories in the region of the seventh layer $(l=7)$, projected onto the 2D plane, exhibiting solid and partial-liquid characteristics. In extended runs we observed that the morphology of the interface fluctuates (on a time scale of $\sim 5 \times 10^{3} \Delta t$ ) between equivalent facet configurations, the one shown in Fig. 1(a) where the facet runs along the $(\overline{1} 10)$ direction and the other one where the facet runs along the (110) direction.

Further insights are provided by the equilibrium particle density profile [Fig. 2(a)] and per-particle potential energies [Figs. 2(b)-2(d)], which show both the crystal-melt and melt-vacuum interfaces. Focusing on
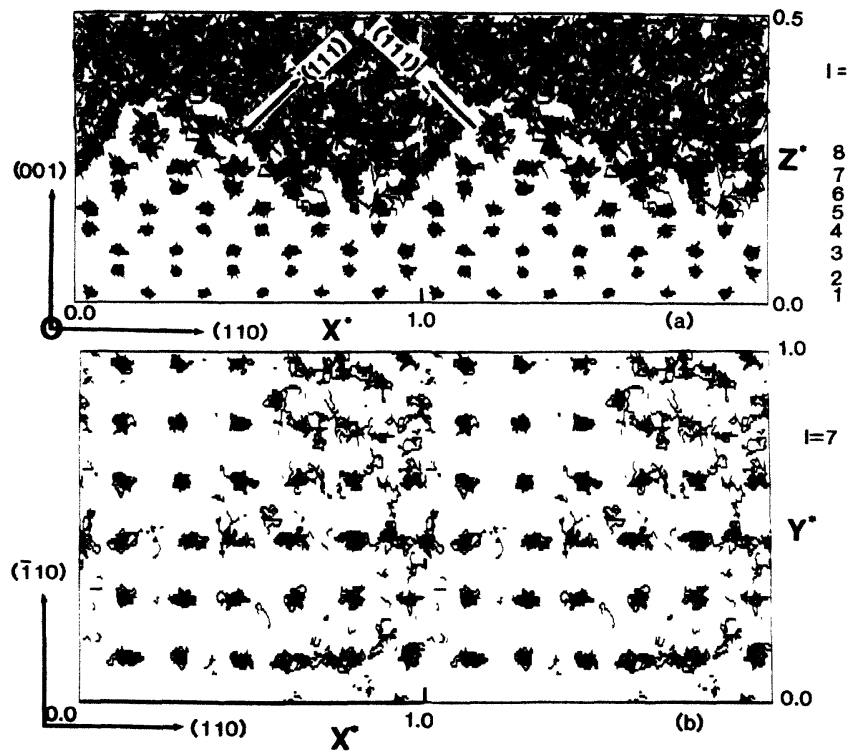

FIG. 1. Real-space particle trajectories at the interface region recorded at equilibrium. (a) Viewed along the [110] direction; (b) trajectories for particles at the region of the seventh layer $(l=7)$ viewed from the [001] direction. $Z^{*}=1 \equiv 18.14 \sigma$ and $X^{*}=Y^{*}=1 \equiv 10.9 \sigma$. The 2D computational cell $\left(0 \leqslant X^{*}, Y^{*} \leqslant 1\right)$ is replicated along the $X^{*}[110]$ direction to aid visualization.

the former interface, we observe the opposing trends in the behavior of the two- and three-body potentials, $V_{2}$ and $V_{3}$, which, when added, yield the result shown in Fig. 2(b). The pronounced minima correspond to the locations of crystalline or partially crystalline layers. We observe that the variation in the total potential energy, $V_{2}+V_{3}$, upon transformation from the solid to the melted region, is smaller than the variation in the individual contributions $V_{2}$ and $V_{3}$. The behavior of the three-body potential can be used to distinguish the solid from the liquid and thus provide a vivid visualization of the solid component of the interface. In Fig. 2(e) contours of $V_{3}$ for $V_{3} \leqslant 0.45$ are shown. By restriction of the value of $V_{3}$, only solidlike regions are captured (the appearance of the $V_{3}$ contour map does not change significantly with small changes in the cutoff value). Figure $2(\mathrm{e})$, in conjunction with the real-space trajectories shown in Fig. 1, complements and corroborates our picture of the structure of the interface.

To affirm further our prediction of the (111) faceting of the equilibrium crystalline $\mathrm{Si}(100)$-melt interface, we present in Fig. 3 results for the Si(111)-melt interface, simulated in a manner similar to that described above, with $N_{L}=20$ and $N_{P}=49$. The particle trajectories viewed along the (110) direction [Fig. 3 (a)] and in-layer trajectories for layers 10,11 and 8,9 , shown in Figs. 3(b) and 3(c), respectively, along with the density and per-particle potential energy profiles in the (111) direction [Figs. 3(d) and 3(e), respectively], 


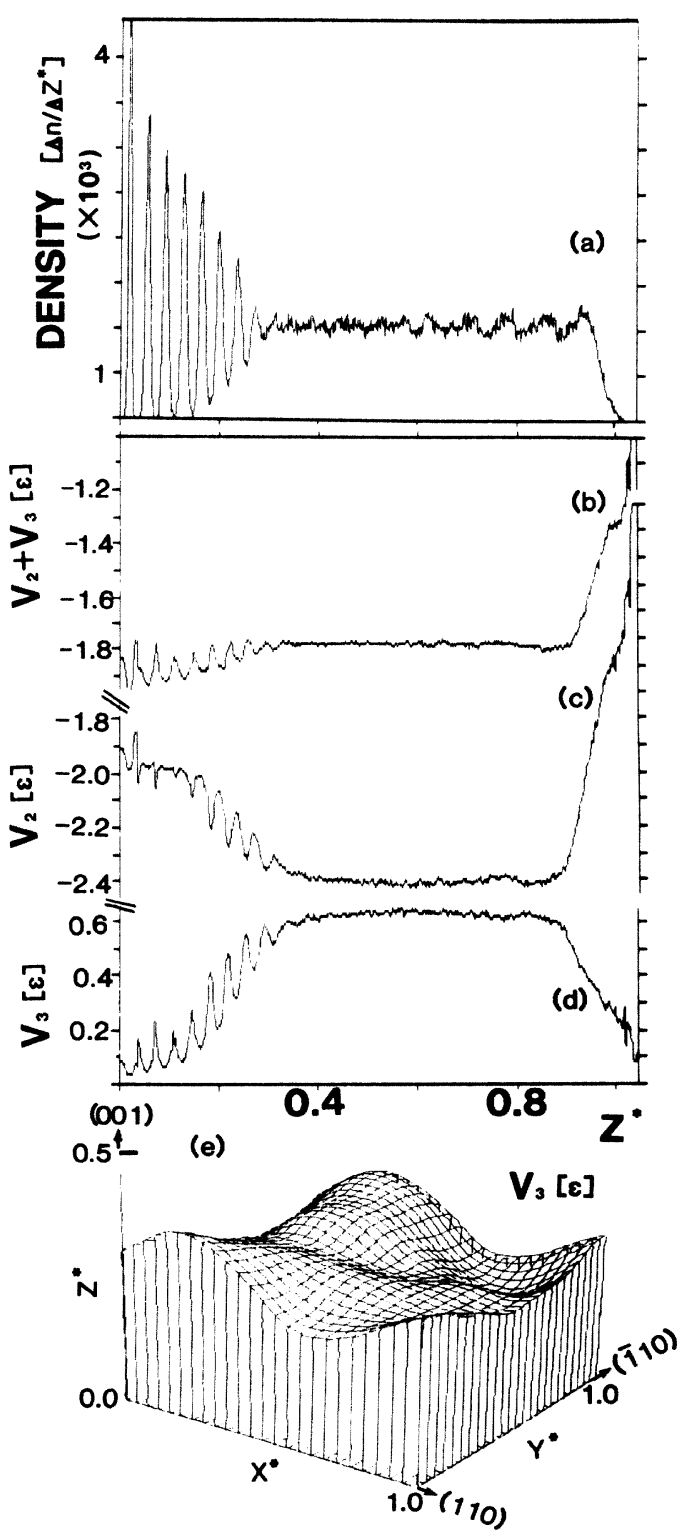

FIG. 2. (a) Equilibrium particle density $\Delta n / \Delta Z^{*}$, where $\Delta n$ is the number of particles with $Z^{*}$ coordinates between $Z^{*}$ and $Z^{*}+\Delta Z^{*}$. (b) -(d) Per-particle potential-energy profiles vs distance along the [001] direction $\left(Z^{*}=1 \equiv 18.14 \sigma\right)$. The total and the two- and three-body potential energies are shown in (b), (c), and (d), respectively. (e) A contour plot of $V_{3}$ for particles with $V_{3} \leqslant 0.45 \epsilon$, exhibiting the solidlike region of the sample, recorded at the same time as the trajectories given in Fig. 1.

provide clear evidence for a sharp, abrupt, and flat $\mathrm{Si}(111)$-melt interface. Additionally, the melt region adjacent to the interface ( $L=10$ and 11$)$ possesses a residual degree of in-planar order.

To compare the predictions of the moleculardynamics simulations with an experimental system, we have observed the solid-melt interface in situ, during melting with a shaped beam of a cw argon laser. The starting material was a $600-\mathrm{nm}$-thick film of polycrys- talline silicon deposited on a quartz wafer by chemical vapor deposition and capped by a layer of silicon dioxide. The sample was mounted on a translation stage positioned upon a vibration-isolated table, and the liquid-solid interface was observed by means of a microscope equipped with a visible and near-infrared video camera. Two laser beams, shaped into elliptical cross sections, and positioned in close spatial proximity, were used to maintain a closely controlled temperature gradient across the molten zone. The size of the molten zone was approximately $25 \times 100 \mu \mathrm{m}^{2}$. After an initial scan to convert the polycrystalline material into a monocrystalline ribbon, the scanning was stopped to establish quasiequilibrium temperature distribution across the molten zone. A view of the solidmelt interface is shown in Fig. 4. The recrystallized film has predominantly (100) texture, as has been confirmed by subsequent $x$-ray diffraction. In the direction normal to the original scan, small periodic deviations from the $\langle 100\rangle$ axis occur, with a characteristic length of approximately $10 \mu \mathrm{m}$. The various sets of facets defined by alternating (111) and (111) planes seen in Fig. 4 appear slightly tilted with respect to each other so as to preserve the relative alignment with the bulk texture. The structure shown in Fig. 4 is typical of many faceted configurations that were observed. The shape of the solid-melt interface fluctuates, as certain facets spontaneously disappear and new facets form. Individual facets typically persist for several video-frame times $\left(\frac{1}{30} \mathrm{~s}\right)$.

In comparison of the experimental and simulation results, the similarity in appearance is rather striking, particularly when one notices that the two differ vastly in spatial and temporal scales. On the foundation of the premise that the origins of all physical phenomena are microscopic in nature, we venture the hypothesis that above a certain size the energetics that govern the structure of the solid-melt interface in this system operate in a similar manner in both the microscopic (theory) and macroscopic (experiment) regimes, and that the dimensions of the morphological characteristics scale with system size. Clearly, the dimensions of the computational cell limit the size of facets that form. For a small facet, the relative number of edge and corner atoms to those located on the facet plane is high, thus affecting its stability. While analysis of the results obtained for the two system sizes which we simulated provides some support to this hypothesis, more theoretical and experimental work is needed. Such theoretical efforts would involve further, extended simulations and the development of improved microscopic theories of interfacial energetics, dynamics, and morphological stability. ${ }^{10}$ On the experimental side, it would be desirable to observe the solid-melt interface on much smaller spatial and temporal scales to record the process of facet formation in greater detail.

The structure of the interface is governed by the en- 


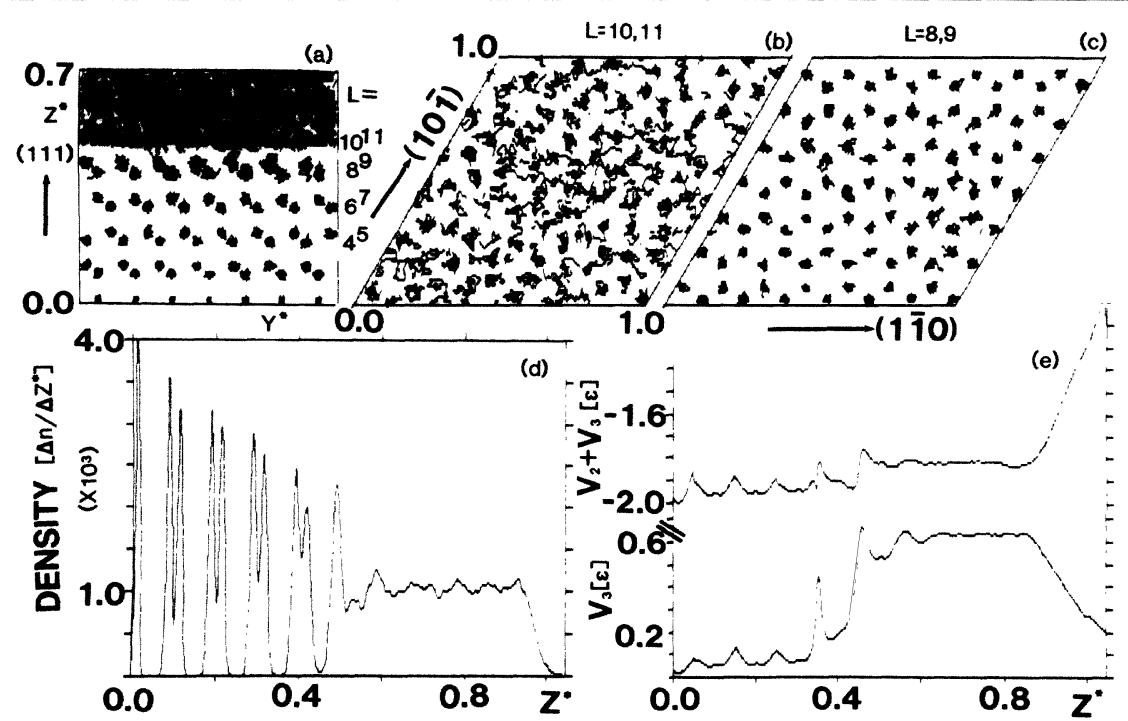

FIG. 3. (a) Particle trajectories recorded at equilibrium, viewed along the [110] direction. (b)-(c) In-plane trajectories, exhibiting solid and melt characteristics, in layers 10,11 and 8,9 , viewed along the $[111], Z^{*}$, direction. $Z^{*}=1=14.96 \sigma$ and the unit of length in the $[1 \overline{1} 0]$ and $[10 \overline{1}]$ directions is $12.85 \sigma$. (d) Equilibrium particle density, $\Delta n / \Delta Z^{*}$, vs $Z^{*}$. (e) Per-particle total and three-body potential energies, $V_{2}+V_{3}$ and $V_{3}$, vs $Z^{*}$.

ergetics, particularly the interplay between the twoand three-body contributions to the potential energy, and by entropic factors. Our observation that faceting initiates upon melting and then further refines upon achieving equilibrium is most likely a result of the fact that the closest-packed (111) planes of silicon are the directions of slowest growth. ${ }^{11}$ In a dynamic equilibrium situation these faces are also the slowest to melt. An important issue in investigations of the recrystallization kinetics of zone-melted silicon is the role of the interface morphology in the generation of low-angle grain boundary defects, whose branching behavior was rather well described by a recent kinetic model, ${ }^{1}$ with the assumptions of a faceted structure of the growth interface and that the growth rate is limited only by the nucleation rate of new monolayers. Having established the faceted equilibrium structure of the interface, including the partial ordering in the nearby melt

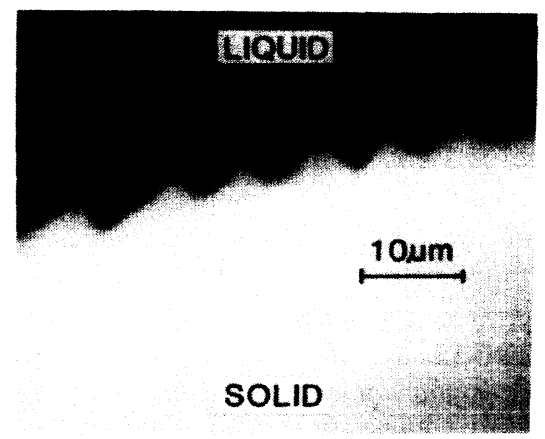

FIG. 4. In situ view (on a video monitor) of the solid-melt interface, in quasiequilibrium, exhibiting (111)-faceted structure region, we expect that further studies will allow us to elucidate the microscopic kinetics and dynamics of growth and subboundary formation.

The theoretical calculations were supported, in part, by U. S. Department of Energy Contract No. EG-S05-5489 and were performed on the Cray-XMP at the the National Magnetic Fusion Energy Computer Center, Livermore, California.

${ }^{1}$ L. Pfeiffer, S. Paine, G. H. Gilmer, W. van Saarloos, and K. W. West, Phys. Rev. Lett. 54, 1944 (1985).

${ }^{2}$ G. K. Celler, K. A. Jackson, L. E. Trimble, Mc. D. Robinson, and D. J. Lischner, in Energy Beam-Solid Interactions and Transient Thermal Processing, edited by J. C. C. Fan and N. M. Johnson (North-Holland, New York, 1984), p. 409.

${ }^{3}$ D. P. Woodruff, The Solid-Liquid Interface, (Cambridge Univ. Press, Cambridge, England, 1973), Chap. 3.

${ }^{4}$ M. W. Geis, H. J. Smith, B.-Y. Tsaur, J. C. C. Fan, D. J. Silversmith, and R. Mountain, J. Electrochem. Soc. 129, 2813 (1982).

${ }^{5}$ K. F. Lee, T. J. Stultz, and J. F. Gibbons, in Semiconductors and Semimetals, edited by R. K. Willardson and A. C. Beer (Academic, New York, 1984), Vol. 17, p. 227.

6(a)F. F. Abraham, J. Vac. Sci. Technol. B 2, 534 (1984).

6(b) U. Landman, R. N. Barnett, C. L. Cleveland, and R. H. Rast, J. Vac. Sci. Technol. A 3, 1574 (1985).

${ }^{7}$ F. H. Stillinger and T. A. Weber, Phys. Rev. B 31, 5262 (1985).

${ }^{8}$ R. N. Barnett, C. L. Cleveland, and U. Landman, Phys. Rev. Lett. 541679 (1985)

${ }^{9}$ M. Parinello and A. Rahman, Phys. Rev. Lett. 45, 1196 (1980).

10J. S. Langer, Rev. Mod. Phys. 52, 1 (1980); R. F. Sekerka, Physica (Amsterdam) 12D, 212 (1984).

${ }^{11}$ G. H. Gilmer, Mat. Res. Soc. Proc. 13, 249 (1983); see also Ref. 6 b. 

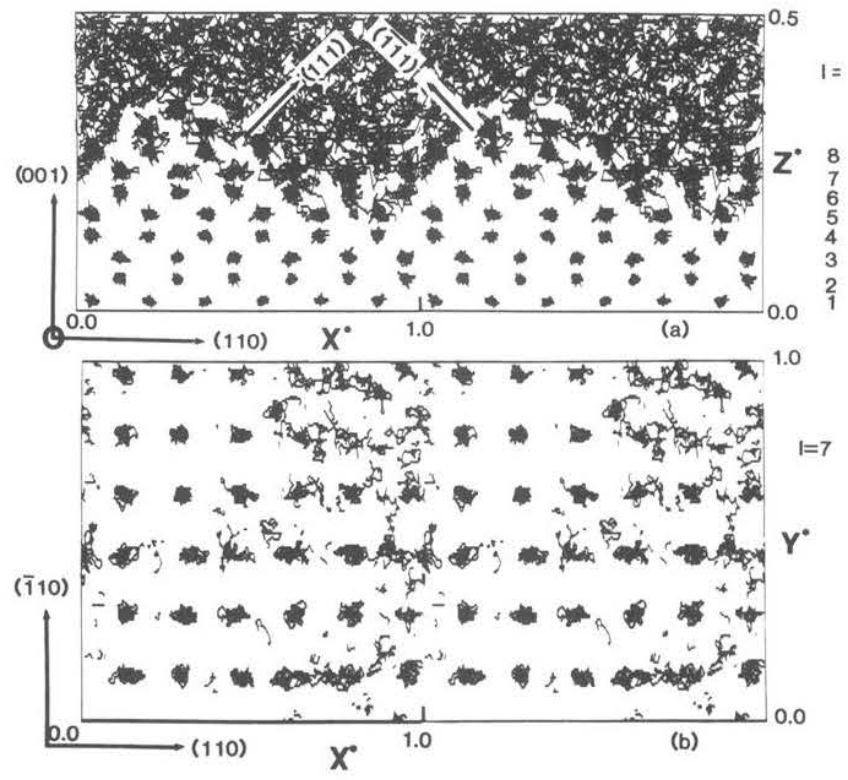

FIG. 1. Real-space particle trajectories at the interface region recorded at equilibrium. (a) Viewed along the [1110] direction; (b) trajectories for particles at the region of the seventh layer $(l=7)$ viewed from the [001] direction. $Z^{*}=1 \equiv 18.14 \sigma$ and $X^{*}=Y^{*}=1 \equiv 10.9 \sigma$. The $2 \mathrm{D}$ computational cell $\left(0 \leqslant X^{*}, Y^{*} \leqslant 1\right)$ is replicated along the $X^{*}[110]$ direction to aid visualization. 


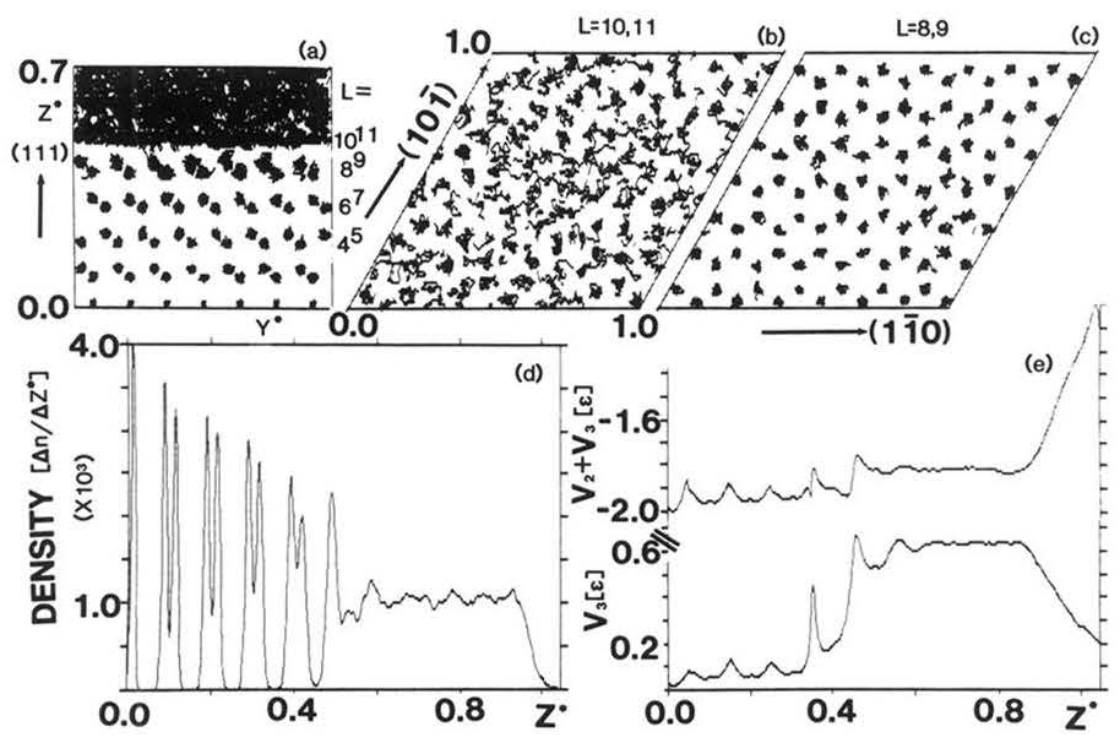

FIG. 3. (a) Particle trajectories recorded at equilibrium, viewed along the [1 100$]$ direction. (b)-(c) In-plane trajectories, exhibiting solid and melt characteristics, in layers 10,11 and 8,9 , viewed along the [111], $Z^{*}$, direction. $Z^{*}=1=14.96 \sigma$ and the unit of length in the [1 $\overline{1} 0]$ and $[10 \overline{1}]$ directions is $12.85 \sigma$. (d) Equilibrium particle density, $\Delta n / \Delta Z^{*}$, vs $Z^{*}$. (e) Per-particle total and three-body potential energies, $V_{2}+V_{3}$ and $V_{3}$, vs $Z^{*}$. 


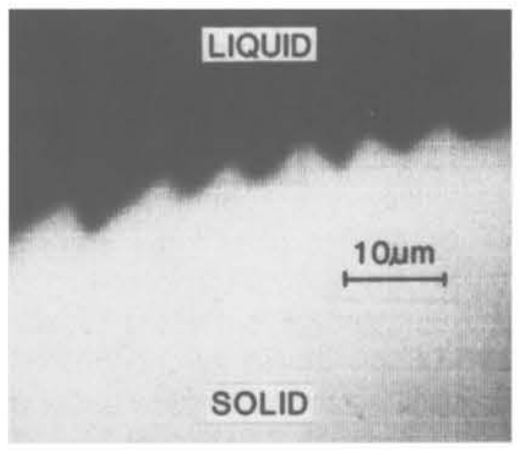

FIG. 4. In situ view (on a video monitor) of the solid-melt interface, in quasiequilibrium, exhibiting (111)-faceted structure. 